\title{
Comparison of DBD plasma actuators flow control authority in different modes of actuation
}

\author{
M. Abdollahzadeh*, J.C. Pascoa, P.J. Oliveira \\ Universidade da Beira Interior, Departamento de Engenharia Electromecanica, C-MAST - Center for Mechanical and Aerospace Sciences and Technologies, Covilha, \\ Portugal
}

\section{A R T I C L E I N F O}

\section{Article history:}

Received 7 September 2017

Received in revised form 1 April 2018

Accepted 6 April 2018

Available online 10 April 2018

\section{Keywords:}

Unsteady actuation

Optimum frequency

Flow control

Analytical model

Resonance mode

\begin{abstract}
A B S T R A C T
The principal mechanisms by which DBD plasma actuators influence flow characteristics, and are thus able to control that flow, depend strongly on their modes of actuation. Here two different modes based on steady and unsteady actuation are compared and investigated. A simple sinusoidal voltage distribution and a duty cycled sinusoidal voltage were considered for these purposes. Leading edge separation around a stalled NACA 0012 airfoil at $\mathrm{Re}=3 \times 10^{6}$ is considered as test case. A simplified phenomenological model which uses the correct scale of the plasma body force is considered for the modeling of the plasma actuator effects. The steady actuation results show that flow control can be effectively achieved by this mode of operation with continuous injection of momentum in the boundary layer. Unsteady actuation with an imposed frequency equal to the calculated natural frequencies of the flow gives rise to a resonance actuation effect.
\end{abstract}

(c) 2018 Elsevier Masson SAS. All rights reserved.

\section{Introduction}

Several passive and active flow control techniques were proposed to manipulate a flow field to improve efficiency or performance with the intention to delay/advance transition, to suppress/enhance turbulence, or to prevent/promote separation [1-4]. The resulting benefits include drag reduction, lift enhancement, mixing augmentation, heat transfer enhancement, and flow-induced noise suppression [5,6]. The use of effective active control flow techniques can provide higher efficiency for the controlled system. Among different Active Flow Control (AFC) techniques, Dielectric Barrier Discharge (DBD) plasma actuators are novel means of actively controlling flow and have gained increased interest during the past decade for different applications [7-12]. This kind of actuators may be operated in different modes depending on the type of the input voltage signal. If a sufficiently high continuous sinusoidal voltage is applied to the electrodes, the plasma actuator will accelerate the fluid. In this mode the main mechanism of flow control is by locally imparting momentum to the nearby flow. The use of a plasma actuator in steady operational mode would cause significant modification of the stability properties in a boundary layer. The amplitudes of the oscillations would tend to decrease. If the voltage source operates in a burst mode, with a specified

\footnotetext{
* Corresponding author.

E-mail addresses: mm.abdollahzadeh@yahoo.com, mm.abdollahzadeh@ubi.pt (M. Abdollahzadeh), pascoa@ubi.pt (J.C. Pascoa), pjpo@ubi.pt (P.J. Oliveira).
}

duty cycle, the momentum injection will be done discontinuously (unsteady mode) with a frequency equal to the frequency of duty cycle. In this mode, the plasma actuator might mitigate or stabilize the natural instabilities of the flow in a more efficient way by generating large coherent vortical structures. In this case, the plasma induced instabilities would counteract the disturbances already present in the flow. The amplitude of the flow instabilities would decrease due to superposition of velocities. In the case of unsteady or pulsed actuation, the power requirement of the actuator is lower than in steady actuation due to the short duty cycles of the input voltage. In fact, the duty cycle percentage correlates linearly with power consumption. In Fig. 1, the schematic of the plasma actuator and the different operational modes of a plasma actuator are shown. In the unsteady mode, the forcing of the actuator during the active period accelerates the flow. When the actuator is turned off periodically, a large vortex forms due to wall effect and decouples from the actuation region. This process is then repeated at every cycle [13].

One of the most common situations in which the importance of flow control is more relevant is the control of flow in stall and post stall condition $[14,15]$. Several studies have been reported in the literature showing the capability of plasma actuators for controlling flow in such situations [16]. To increase the efficiency of the plasma actuators, some of the relevant parameters can be optimized to reach higher performances $[17,18]$. Chernyshev et al. [19] proposed a simple new design for a multiple-DBD actuator intended for flow control in a thin boundary layer on a lengthy 


\section{Nomenclature}

c Chord length $\ldots \ldots \ldots \ldots \ldots \ldots \ldots \ldots \ldots \ldots \ldots \ldots \ldots \ldots \ldots \ldots, m$

$C_{L} \quad$ Lift coefficient

$C_{D} \quad$ Drag coefficient

E Electric field $\ldots \ldots \ldots \ldots \ldots \ldots \ldots \ldots \ldots \ldots, \mathrm{V} / \mathrm{m}$

$f \quad$ Frequency of applied voltage $\ldots \ldots \ldots \ldots \ldots \ldots, \mathrm{Hz}$

$f(t) \quad$ Voltage shape (in Eq. (3))

$F_{\beta} \quad$ Burst frequency $\ldots \ldots \ldots \ldots \ldots \ldots \ldots \ldots \ldots, \mathrm{Hz}$

$$
F_{\beta}=1 / T_{\text {signal }}
$$

$F^{+} \quad$ Reduced burst frequency, $F^{+}=F_{\beta} c / U_{\infty}$

$l, t_{e}, t_{d} \quad$ Length and thickness of electrode or dielectric .... m

$\operatorname{Re} \quad$ Reynolds number, $\operatorname{Re}=\frac{U_{\infty} c}{v}$

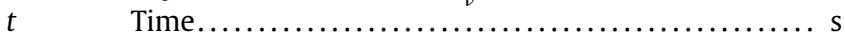

$U_{\infty} \quad$ Free stream velocity $\ldots \ldots \ldots \ldots \ldots \ldots \ldots \ldots \ldots \ldots \ldots, \mathrm{m} / \mathrm{s}$

M Mach number

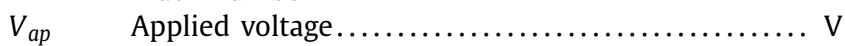

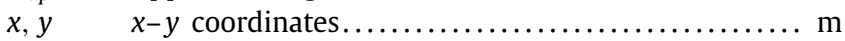

$x^{\prime} \quad$ coordinate along the chord of airfoil $y^{\prime} \quad$ coordinate normal to the chord of airfoil

Greek symbols

$\beta \quad$ Burst ratio, $\beta=T_{\text {control }} / T_{\text {signal }}$

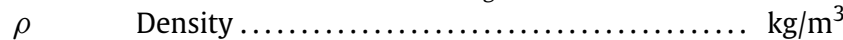

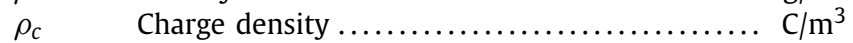

$v \quad$ Kinematic viscosity $\ldots \ldots \ldots \ldots \ldots \ldots \ldots \ldots \ldots \mathrm{m}^{2} / \mathrm{s}$

$\alpha \quad$ Free stream flow angle

$\varepsilon \quad$ Dielectric permittivity

$\phi \quad$ Electric potential ............................. V

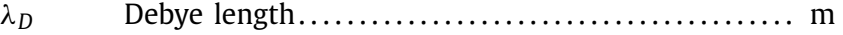

\section{Subscripts}

Sep Separation

Sl Shear layer

Shed Shedding

ap applied
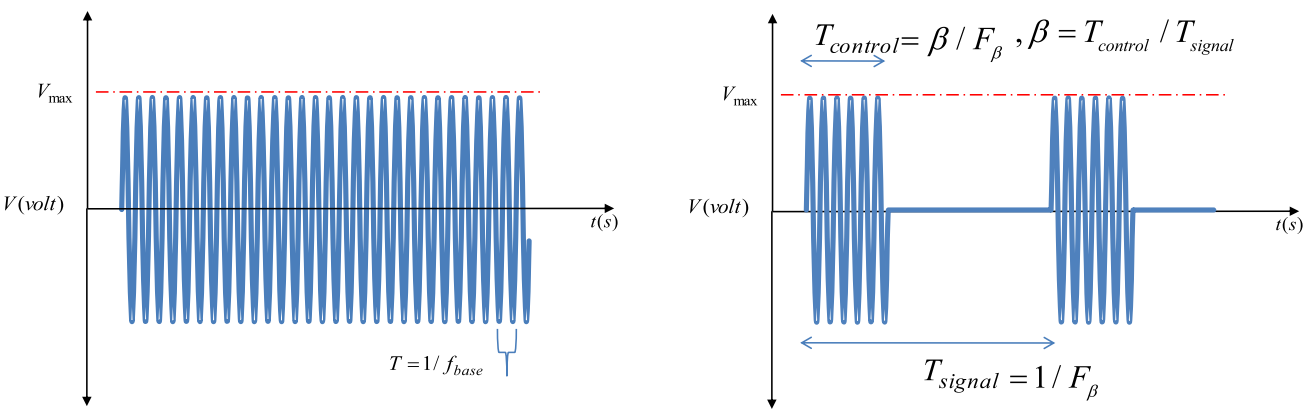

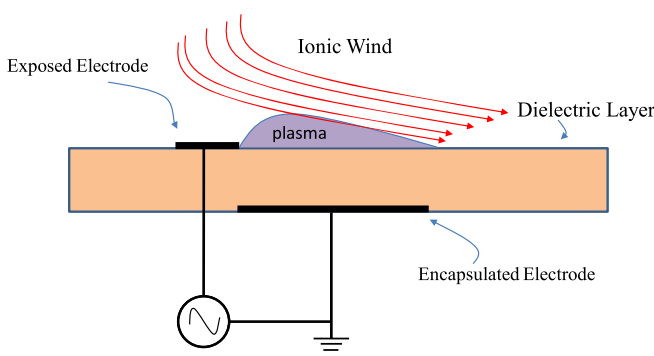

(a)

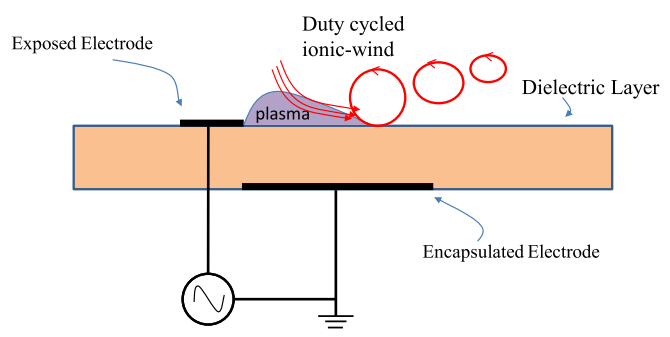

(b)

Fig. 1. a) plasma actuator in steady operation mode; b) plasma actuator with duty cycle applied voltage.

surface. Erfani et al. [20] conducted an optimization study of a multi-encapsulated electrodes design of DBD plasma actuators in order to produce higher velocities providing more momentum into the background air. Tsubakino and Tanaka [21] have shown that the location of the plasma actuator has an influence on the flow control capability. In our previous work [22], we have shown that, for optimum flow control, plasma actuator should be placed before the separation point. The shape of the voltage wave has also an influence on the electric discharge and thus on the trust generated by plasma actuators [23-25]. In the case of unsteady actuation, the burst ratio or duty cycle $\left(\beta=T_{\text {control }} / T_{\text {signal }}\right)$ and the modulation or burst frequency $F_{\beta}=1 / T_{\text {signal }}$ (see Fig. 1 ) are the major factors that may influence the efficiency of the plasma actuator [26]. The burst ratio $\beta$ is the temporal duration of the actuation $\left(T_{\text {control }}\right)$ divided by the period of one burst cycle $\left(T_{\text {signal }}\right)$. A burst ratio of $100 \%(\beta=1.0)$ means the steady actuation mode.
Asada et al. [27] have studied experimentally the effect of burst ratio and burst frequency. They concluded that smaller burst percentage allows increased flow control capability and at higher duty cycle frequency the voltage needed for controlling the flow is smaller and thus higher burst frequencies are more efficient. They have also shown that the reduced frequency of $F^{+}=9.1$ is optimal for flow control. Similar results have been reported by Audier et al. [28]. Patel et al. [29], reported that the optimum frequency of unsteady actuation at which the voltage needed to reattach the flow is minimum is equal to $F^{+}=1\left(F^{+}=F_{\beta} c / U_{\infty}\right)$. To obtain the optimum frequency, the actuator voltage was varied until a sudden rise in the lift coefficient was observed for the specified frequency. Moreover, they mentioned that if the burst frequency is not optimal, the effect of the plasma actuator would be negligible, and showed that the minimum voltage to control the flow varies with the angle of attack and flow velocity. In the experiments conducted by Sidorenko et al. [30], the existence of an 
optimum frequency for both pulsed voltage and burst modes of actuation was observed $\left(F^{+}=2.6-14\right)$. Mitsuo et al. [31] showed that flow control authority of the plasma actuator is sensitive to the frequency of the unsteady plasma actuation. However, their measurements showed a value around $F^{+}=0.5$ for the optimum burst modulation frequency. Benard et al. [32] studied experimentally the effect of steady and unsteady actuation on the lift and drag coefficients. Their results showed an aerodynamic enhancement when the frequency coincides with natural vortex frequency. Furthermore, Benard and Moreau [33], explored the use of multifrequency excitation voltage signal to cover a wide range of the frequency of the flow instabilities. These studies suggest that there exists an optimum point for the parameters involved in unsteady or burst mode of actuation. Akansu et al. [34], experimentally investigated the effect of four DBD plasma actuators on the manipulation of separation over a NACA0015 airfoil at low Reynolds number. They studied several parameters including the effects of applied voltage amplitude, dielectric thickness and unsteady actuation. Another situation for which periodic force generation by plasma actuators would benefit flow control is the dynamic stall, where unsteady plasma actuation enhances the lift and production of concentrated vortex structure near the leading edge [35].

Although most of these studies report an optimum value of $F^{+}=1$ for the bust frequency, there are still discrepancies between the values suggested by some studies. The objective of the present paper is to investigate and compare the flow control ability of DBD plasma actuators under different modes of actuation. All of these modes of actuation were tested with only one single DBD actuator to control the leading-edge separation of the airfoil, at stall condition. Since plasma actuation in unsteady mode could influence the stability of the boundary layer and turbulization (higher frequency), or the instabilities of the separation layer and shedding structure (lower frequency), we have applied FFT analysis on the velocity components in order to obtain the natural frequencies of the flow. Then, for the unsteady mode of actuation, different values of the burst frequencies were tested including the natural frequencies of flow instabilities.

\section{Plasma effect modeling}

Recently, we have presented a simple phenomenological model based on the scaling of the plasma generated thrust which allows us to compute the body force in the momentum equation due to plasma actuation [36]. The model uses the Gauss law to obtain the electric field distribution, in conjunction with an experimentally verified distribution of the particles charge density over the surface of the dielectric material for obtaining the body force. The governing equations of the model (electric potential and charge density) are as follows:

$\nabla \bullet(\varepsilon \nabla \phi)=0$

$\nabla \bullet\left(\varepsilon_{r} \nabla \rho_{c}\right)=\frac{\rho_{c}}{\lambda_{D}^{2}}, \quad \varepsilon=\varepsilon_{0} \varepsilon_{r}$

These equations do not contain a time derivative term. It is only the boundary condition for the applied voltage at the exposed electrode and for the charge density at the dielectric surface which are time dependent. Therefore, Eqs. (1) and (2) can be normalized as follows for a two-dimensional coordinate system:

$$
\begin{aligned}
\rho_{c}^{*} & =\frac{\rho_{c}}{\rho_{c, \max } f(t)}, \quad \phi^{*}=\frac{\phi}{\phi_{\max } f(t)}, \\
\vec{E}^{*} & =\nabla \phi^{*}=l_{p}\left(\frac{\partial \phi^{*}}{\partial x} \vec{i}+\frac{\partial \phi^{*}}{\partial y} \vec{j}\right),
\end{aligned}
$$

where

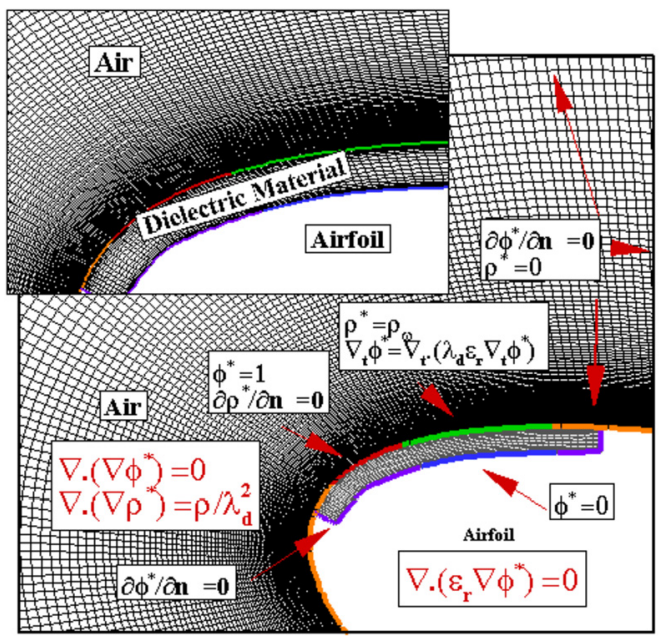

Fig. 2. Schematic of the DBD configuration near the leading of the airfoil and the corresponding boundary conditions for the plasma model.

$\rho_{c, \max }=2 f(t) C_{e q} \frac{\left(V_{a p p}-V_{b d}\right)}{f_{c o r r} \lambda_{d}}$,

$f_{\text {corr }}=\frac{1}{2} \sqrt{2 \pi} \frac{\sigma}{l_{p}}\left[\operatorname{erf}\left(\frac{1}{2} \frac{\mu \sqrt{2}}{\sigma}\right)+\operatorname{erf}\left(\frac{1}{2} \frac{\sqrt{2}\left(l_{p}-\mu\right)}{\sigma}\right)\right]$

$\vec{E}=E_{0} \vec{E}^{*} \quad E_{0}=\frac{\left(V_{a p p}-V_{b d}\right)}{l_{p}}$

In the above equations $f(t)$ is a function representing the shape of the applied voltage, $V_{b d}$ is the breakdown voltage, $l_{p}$ is the length of the plasma region, $\mu$ is the location parameter, $\sigma$ is the scale parameter and $\vec{E}^{*}$ is the normalized electric field vector. Once the dimensionless distribution is determined, the dimensional values at any given time can be obtained by multiplying this distribution with the corresponding normalization factor. In this manner, there is no need to solve the plasma model in an unsteady manner. The body force generated by the plasma actuator depends on the charge density $\left(\rho_{c}\right)$ and the electric field $(\vec{E})$ as expressed by,

$\vec{F}_{0}=\rho_{c} \vec{E}$

The normalized governing equations and the corresponding boundary conditions for the plasma model are summarized in Fig. 2 for the considered DBD actuator geometry.

\section{Problem statement and numerical procedure}

The test case consists on the flow around a NACA 0012 airfoil with chord length of $1 \mathrm{~m}$ under atmospheric conditions. The Reynolds number based on the chord length and the free stream velocity equals $3 \times 10^{6}\left(U_{\infty}=43.81 \mathrm{~m} / \mathrm{s}\right)$. A single DBD plasma actuator is placed at the leading edge of the airfoil, mostly for controlling the stall. The exposed electrode length is $15.47 \mathrm{~mm}$ and it starts at $x / c=0.001481$. The embedded electrode is $26.57 \mathrm{~mm}$ long and its leading edge is placed at $x / c=0.01149$. Both electrodes were assumed to have thickness of $70 \mu \mathrm{m}$. The embedded electrode is covered with thin layer of dielectric material which is $4 \mathrm{~mm}$ thick and has a dielectric permittivity equal to $\varepsilon=4 \varepsilon_{0}$. A schematic of the electrodes and dielectric layer configuration is presented in Fig. 2. Although the plasma model makes use of the thickness of the electrode for estimating the thrust induced by the plasma actuator, the numerical grids were built assuming that electrodes are infinitely thin layers.

Two dimensional unsteady incompressible Navier-Stokes equations are considered for obtaining the flow field variables (velocity and pressure) and are written in tensor form as: 
$\frac{\partial u_{i}}{\partial x_{i}}=0$
$\frac{\partial u_{i}}{\partial t}+\frac{\partial u_{i} u_{j}}{\partial x_{j}}=-\frac{1}{\rho} \frac{\partial p}{\partial x_{i}}+\frac{1}{\rho} \frac{\partial \tau_{i j}}{\partial x_{j}}+f_{i}$

In the above equations, the indices $i=1,2$, represent respectively the $x$ and $y$ direction, $p$ is the pressure, $\mu$ is dynamic viscosity, $t$ is the time, $\tau_{i j}$ is shear stress tensor, $u_{i}$ is the $i$-component of the velocity vector and $f_{i}$ is the component of the body force vector per unit mass arising from the plasma model $(\vec{f}=\vec{F} 0 / \rho)$.

Numerical solution of the governing equations stated above and in the previous section was accomplished by discretizing them with a finite volume approach, using a cell centered collocated arrangement of primitive variables. The numerical implementation was conducted by developing a new solver in OpenFoam [37]. All the convective fluxes were approximated by the GAMMA scheme using face center values for velocity. It is known that GAMMA introduces somewhat more dissipation in regions of high gradients compared with other more sophisticated high-resolution schemes, or central differences, but it allowed us to obtain iterative convergence when the other schemes failed. The pressure-velocity coupling is solved by the PISO algorithm and time is advanced with a second-order backward-differencing scheme. In simulating the procedure of different frequencies of unsteady excitation, the time step is kept in the order of $10^{-5} \mathrm{~s}$, with 50 sub-iterations, so that there are sufficient discrete time steps in each period of excitation (for the maximum burst frequency of $1 \mathrm{kHz}$ ). Although the predictability of the URANS and Hybrid LES-RANS models for the case of unsteady actuation needs to be verified $[38,39]$, here we have used those methods together with $k-w$ SST turbulence model [40] and a scale-adaptive simulation technique (SAS) [41]. The uncontrolled flow is a multi-frequency system, which possesses different major frequencies including the frequency of shear layer, frequency of separation bubble and frequency of vortex shedding. Since URANS models may filter out fluctuations above some specified frequency level (e.g. that of the shear layer), when calculating natural frequencies of the flow, the SAS model had been used. A C-type computational grid was created and the grid spacing was refined near the airfoil surface to assure the condition $y+<1$ for correctly capturing the boundary layer development. Different grid densities have been tested for assuring that the results obtained are independent of the numerical grid. The boundaries representing the free stream conditions were considered far enough from the airfoil ( $15 c$ ahead of the airfoil and $20 c$ at the back).

The solution procedure consists of two main steps. The first step is to solve the normalized form of the governing equations for the plasma model in order to obtain the magnitude and distribution of the body force generated by the plasma actuator. In the second step the normalized body force was introduced as an explicit source term in momentum equations which are solved in a sequence fashion with the continuity equation in order to obtain the velocity and pressure fields. The convergence of the numerical procedure is assured by reaching a minimum relative error of $10^{-6}$ at each time step for all variables. An example of the computational grid and the corresponding boundary conditions are shown in Fig. 3.

\section{Results and discussion}

In the sections below, we first present the validation results for the flow with the plasma off and give the main physical features of the DBD. We then do a power-spectral study of that flow to determine the main frequencies at play which may after be used to impose locking conditions in unsteady actuation. After that we give and discuss the results for the steady mode of the DBD actuation, followed by the unsteady mode which is probed in more

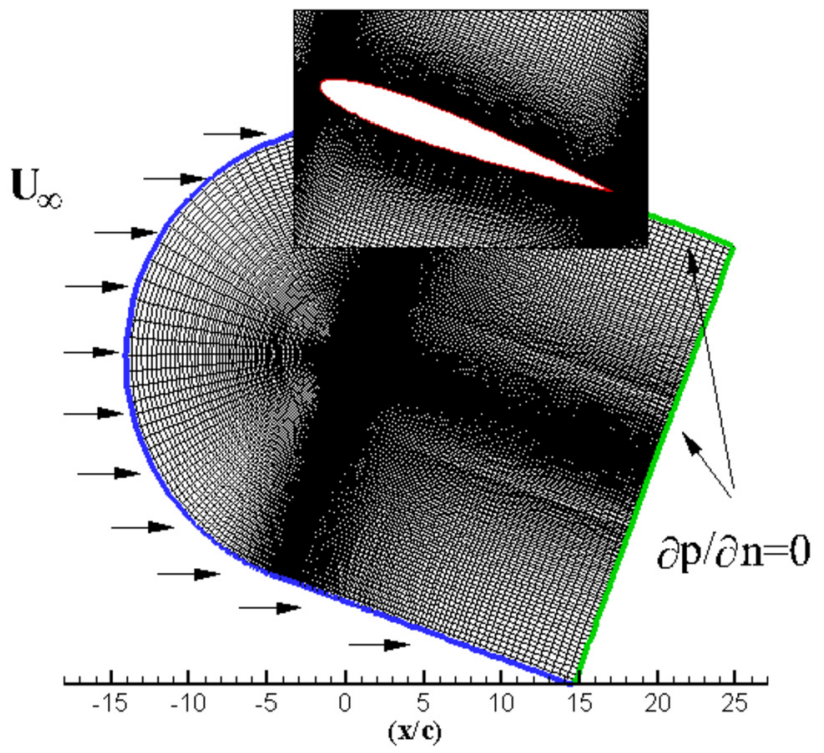

Fig. 3. The computational grid and boundary conditions for the flow field.

\begin{tabular}{lc} 
Table $\mathbf{1}$ \\
Configuration of the single DBD actuator. \\
\hline$V_{a p}$ & $15 \mathrm{kV}$ \\
$f_{\text {ext }}$ & $15 \mathrm{kHz}$ \\
$t_{d}$ & $4 \mathrm{~mm}$ \\
$\varepsilon_{d}$ & 4 \\
$l_{\text {exposed }}$ & $15.47 \mathrm{~mm}$ \\
$l_{\text {embedded }}$ & $26.57 \mathrm{~mm}$ \\
$t_{e}$ & $4 \mathrm{~mm}$ \\
\hline
\end{tabular}

detail (influence of $F_{\beta}$ and $\beta$ on the $C_{p}, C_{L}$ and $C_{D}$ variations are presented).

\subsection{Validation and main DBD characteristics}

For the purpose of validation, the results of the present simulations without the effect of the plasma actuator are compared with the experimental study of Ladson [42]. When there is no actuation applied on the surface of the airfoil, the onset of the stall is around 16 degrees. Above this angle of incidence, a large separation bubble is formed and the lift coefficient drops sharply. Fig. 4a illustrates the flow structure at an angle of $20^{\circ}$ without actuation and Fig. 4b compares the results of the present study for the lift coefficient with the experimental results of Ladson. It is clearly that the present numerical modeling approach provides acceptable accuracy in predicting the flow except for a small discrepancy when stall is approached.

For the purpose of controlling the flow, a single DBD actuator with the configuration specified in Table 1 was applied. A highvoltage continuous sinusoidal signal of $15 \mathrm{kV}$ with frequency of $15 \mathrm{kHz}$ was used for actuation. It should be mentioned that this configuration might not be the optimum for the actuation process.

When a steady actuation is applied, the fluid above the actuator is accelerated and the so-called ionic wind is formed. This accelerated flow region will extend along the airfoil surface. The maximum value of velocity obtained by plasma actuation is around $18.5 \mathrm{~m} / \mathrm{s}$ and the core of the region of accelerated flow is located at $x / c=0.02699$, which is after the separation point for the angle of attack of $20^{\circ}(x / c=0.020022)$. The ionic wind will impart momentum to the flow around the location of the actuator and will tend to stabilize the nearby flow fluctuations and promote reattachment of the boundary layer. Fig. 5 illustrates these features by 


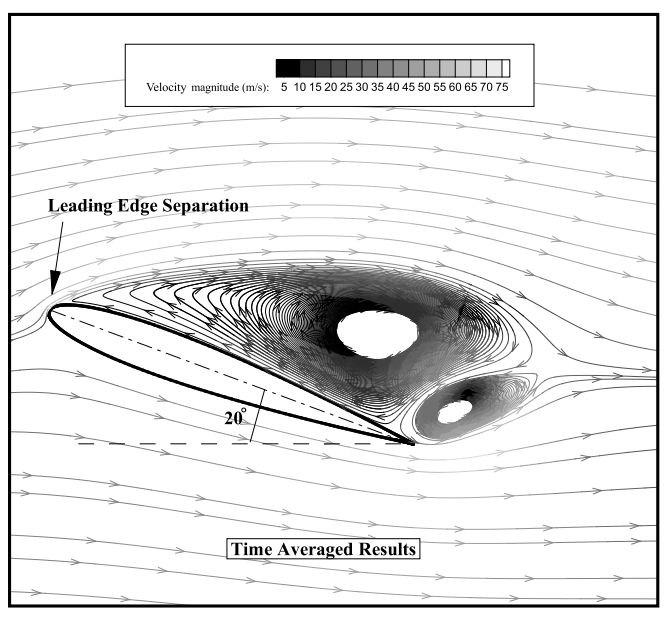

(a)

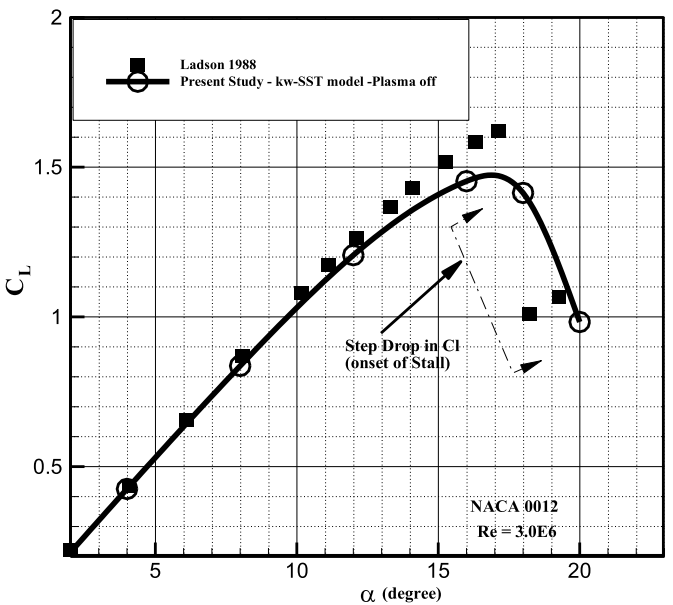

(b)

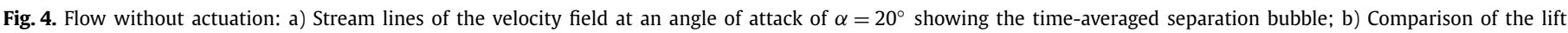
coefficient $\left(C_{L}\right)$ obtained from the present study and experimental results of [42].

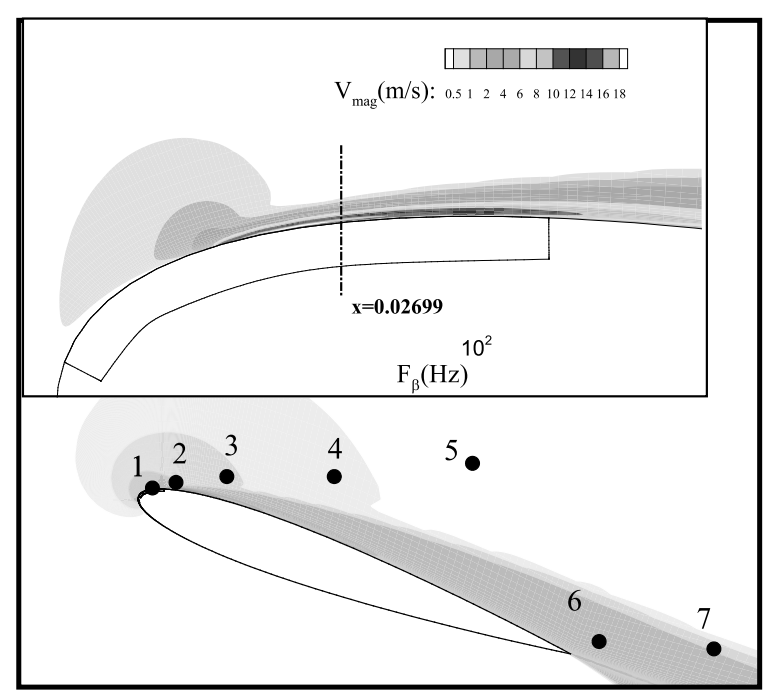

Fig. 5. Velocity contours of the plasma ionic wind at an angle of attack of $\alpha=20^{\circ}$ in quiescent air.

showing velocity contours of the ionic wind formed due to the steady operation of the DBD actuator.

\subsection{Analysis of dominant flow frequencies}

Since the choice of the applied DBD signal and burst frequencies is closely related to the dominant natural frequencies of the base flow, in particular to achieve an effective controlled actuation, we analyze here those dominant frequencies. At the specified incidences, when separation occurs over the airfoil surface three different frequency scales are identifiable. Existence of all these frequencies depends on whether the separated shear layer will reattach before the trailing edge. These frequencies may be classified according to their origin: frequency of the shear layer $f_{S l}$; frequency of the separation bubble $f_{\text {sep }}$; and frequency of global instability $f_{\text {shed }}$ which is related to vortex shedding. They can be scaled as $f_{S l} \approx \frac{\bar{U}}{\theta}, f_{\text {sep }} \approx \frac{U_{\infty}}{L_{\text {sep }}}$ and $f_{\text {shed }} \approx \frac{U_{\infty}}{L_{\text {wake }}}$ where $\bar{U}$ is average velocity in the shear layer, $\theta$ is the momentum thickness at the separation point, $L_{s e p}$ is the length of separation bubble, and $L_{\text {wake }}$ is the characteristic width of the wake. Using the results of the simulations without actuation (therefore the flow is
Table 2

\begin{tabular}{lll}
\multicolumn{2}{l}{ Velocity sensors monitoring locations. } \\
\hline Probe number & $x(\mathrm{~m})$ & $y(\mathrm{~m})$ \\
\hline 1 & 0.0300 & 0.0187 \\
2 & 0.0812 & 0.0308 \\
3 & 0.1918 & 0.0435 \\
4 & 0.4255 & 0.0435 \\
5 & 0.7264 & 0.0723 \\
6 & 1.0018 & -0.3152 \\
7 & 1.2515 & -0.3312 \\
\hline
\end{tabular}

that of Fig. 4a), the characteristic frequencies of the flow were determined by computing power spectra of time-series of $v$-velocity in the shear layer, the separated region, and the wake, using FFT analysis. For this purpose, seven probe locations were considered for monitoring the variation of the velocity components. The locations of these points are tabulated in Table 2, with the origin of the $x, y$ coordinates at the leading edge location $(0,0)$, and are shown by the dots in Fig. 5. Those points are not shown in Fig. 4a because they would be hidden by the stream lines. The basis for the choice of those locations is that points 1,2 and 3 are in the shear layer, points 4 and 5 in the separation bubble, and points 6 and 7 in the shedding wake region.

In Fig. 6 , the variation of the cross-stream velocity ( $v$-component) at different probe locations for the angle of attack of $20^{\circ}$ is shown along with the corresponding power spectra (PS). It is noted that the frequency of shear-layer instability cannot in principle be captured by the numerical simulation using URANS. Firstly, because the time step may filter out the fluctuations of that level, and secondly because using RANS might distort the correct spectral content of the signal (as an outcome of the RANS averaging procedure). Typically, the URANS approach can provide accurate results in situations where the rate of the time variation of the flow has much lower frequency than the turbulence. To overcome this issue, the SAS technique was here applied. As can be seen from Fig. 6, the shear layer, separation bubble and the wake are all locked on a major dominant frequency which is equal to $22.3 \mathrm{~Hz}$. However, two other characteristic frequencies are also observed, one around $3.6 \mathrm{~Hz}$ and the latter around $45 \mathrm{~Hz}$. Since these frequencies are less energetic, they may possibly be some sub/super harmonics of the main natural frequency. The reduced/normalized frequency can then be calculated using the scales introduced above, and the resultant reduced frequencies are presented in Table 3. The value of 

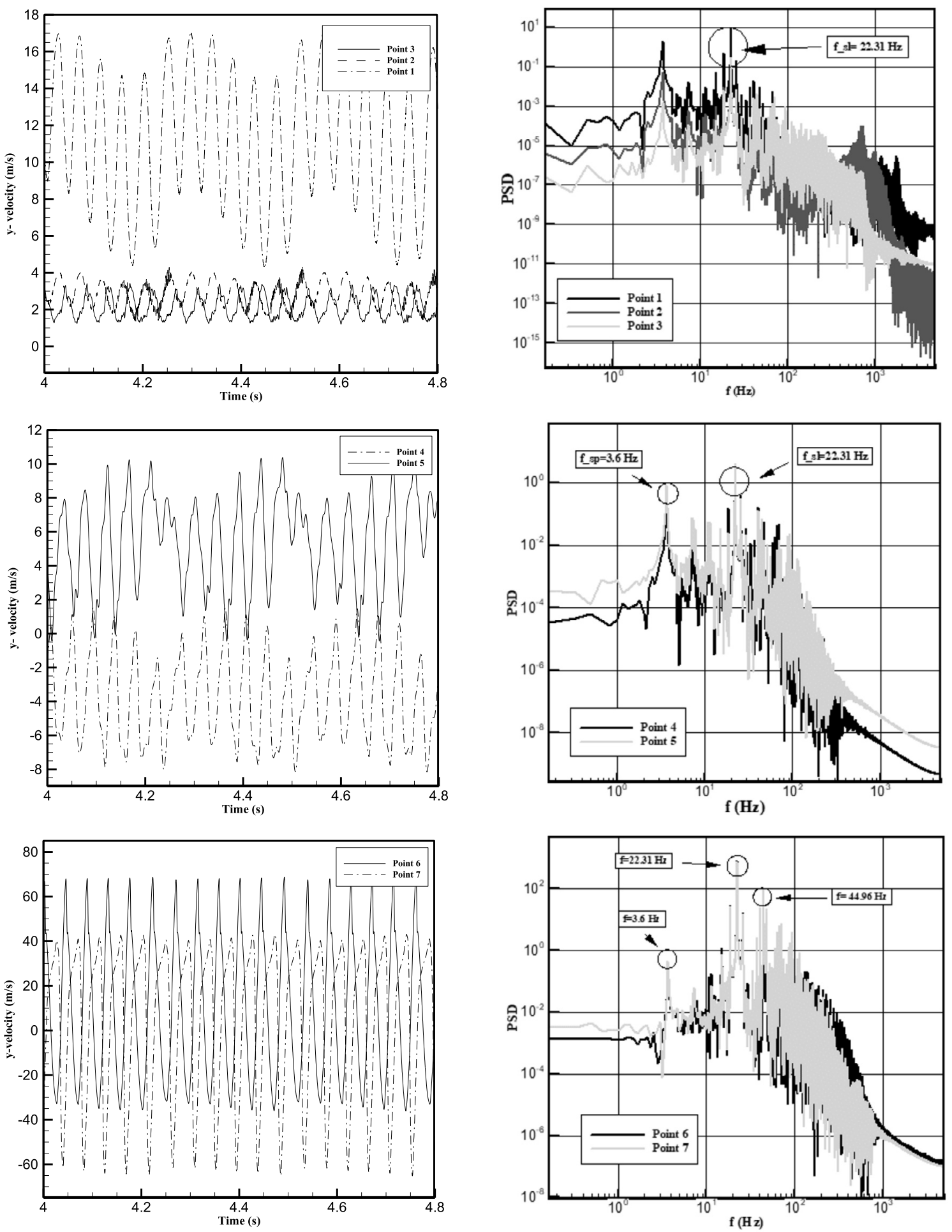

(a)

(b)

Fig. 6. Cross-stream velocity component signals without actuation at different probe locations (given in Fig. 5): a) time variation; b) power spectra.

the reduced shedding frequency of 0.175 (often referred to as the Strouhal number) is in the range reported in literature [43].

\subsection{Results with DBD actuation-steady mode}

Since the plasma actuators were placed slightly upstream of the separation point so as to have an optimum controlling effect, they can be used either in steady mode for stabilization, or in an unsteady manner to lock on the major frequencies of the flow and
Table 3

Dominant normalized frequencies of the flow without actuation.

\begin{tabular}{lll}
\hline $\begin{array}{l}\text { Measured frequency } \\
f(\mathrm{~Hz})\end{array}$ & $\begin{array}{l}\text { Normalized } \\
f_{\text {sep }}^{+}=\frac{f_{\text {sep }}}{U_{\infty}}\end{array}$ & $\begin{array}{l}\text { Normalized } \\
f_{\text {shed }}^{+}=\frac{f_{\text {shed }} c \sin \alpha}{U_{\infty}}\end{array}$ \\
\hline 3.6 & 0.082 & 0.0281 \\
22.3 & 0.509 & 0.1740 \\
45 & 1.027 & 0.3513 \\
\hline
\end{tabular}




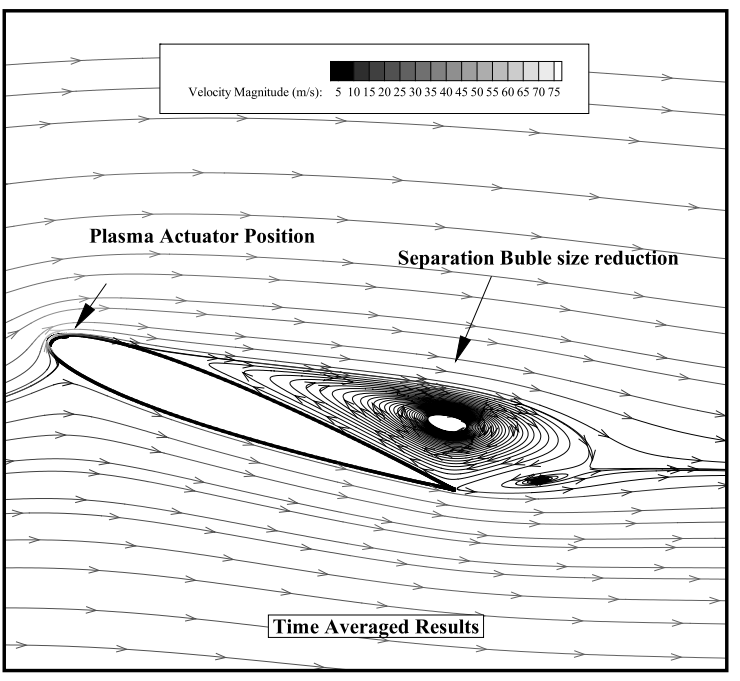

(a)

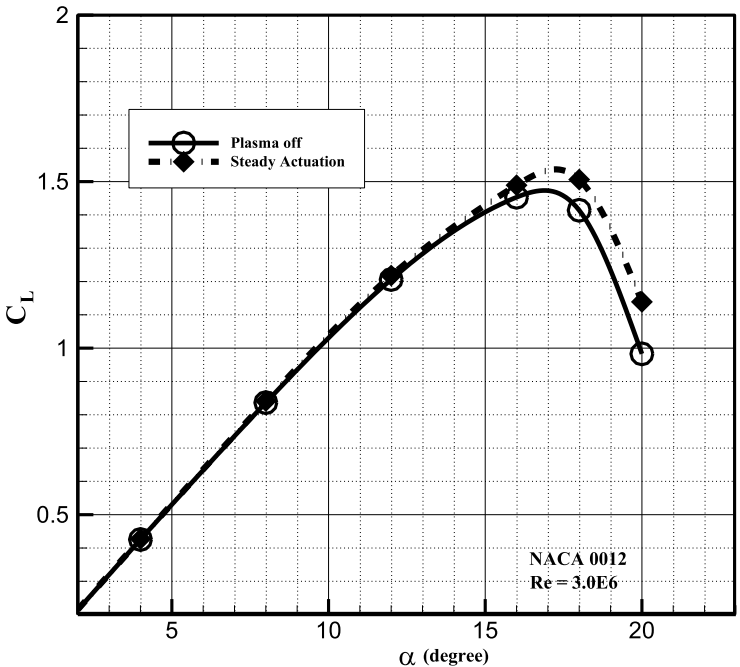

(b)

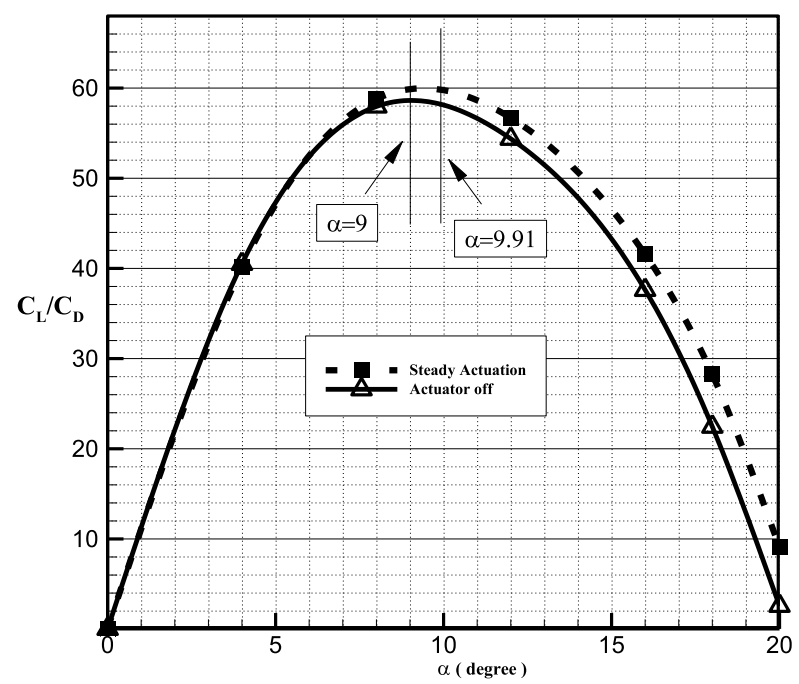

(c)

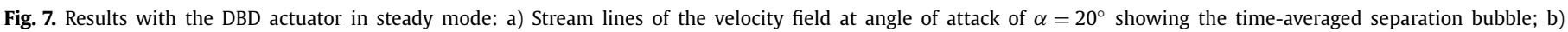
enhancement of the lift coefficient $\left(C_{L}\right)$ by actuation; c) effect of steady plasma actuation change on the pick aerodynamic performance point.

their harmonics, so as to initiate a hydrodynamic resonance or optimum controlling effect. In Fig. 7a, the time averaged velocity stream lines reveal the significant reduction of the size of the separation bubble in the case of steady actuation, for the angle of attack of 20 degrees. Figs. 7b and 7c compare the variation with $\alpha$ of lift coefficient and the lift/drag ratio of the airfoil $C_{L} / C_{D}$ for the cases with and without actuation. These results show that the steady operation of the DBD actuator successfully improves the lift coefficient of the airfoil. At high angle of attack, the increment of $C_{L}$ is more pronounced when stall occurs. At the angle of attack $20^{\circ}$, the lift coefficient was increased approximately by $16 \%$ with its value raising from 0.99 to 1.16 . Moreover, the reattachment of the flow decreases the effective angle of attack of the airfoil and, therefore, improves the aerodynamic performance of the airfoil (Fig. 7c).

Fig. 8 illustrates the changes brought about to the velocity fluctuations and the corresponding power spectra when the steady actuation is applied. As can be seen, when the plasma actuator is working in steady mode, all the major frequencies of the flow vanish and the flow becomes more stabilized. There is also a reduction in the power spectrum peak, and regularization of the fluctuations indicating that possibly noise reduction might be achievable by using the DBD plasma control.

\subsection{Results with DBD actuation-unsteady mode}

In the case of unsteady actuation with alternating current (AC), reduced frequencies in the range 0.1 to 23 , with two burst modulations of $10 \%$ and $50 \%$, were tested for possible observation of resonance behavior. Due to the actuation, ionic wind is formed during the active part of the cycle. When the actuation stops, the plasma-induced vortex separates from the actuation location and interacts with the main flow separation bubble and thus allowing some control of the flow. In Fig. 9, the time averaged streamlines are superimposed on velocity field contours and are shown at three burst frequencies and two burst ratios $(\beta=10 \%$ on the left and $\beta=50 \%$ on the right). The shape of the separation bubble varies significantly with the burst frequency and burst ratio. The size of the separation bubble is reduced when $\beta=50 \%$ for all the burst frequencies employed here. The center of circulation region moved further downstream when the frequency is increased from $f=25 \mathrm{~Hz}$ to $f=250 \mathrm{~Hz}$. In the case of $\beta=10 \%$, the suppression of the separation bubble was not significant for the lower 

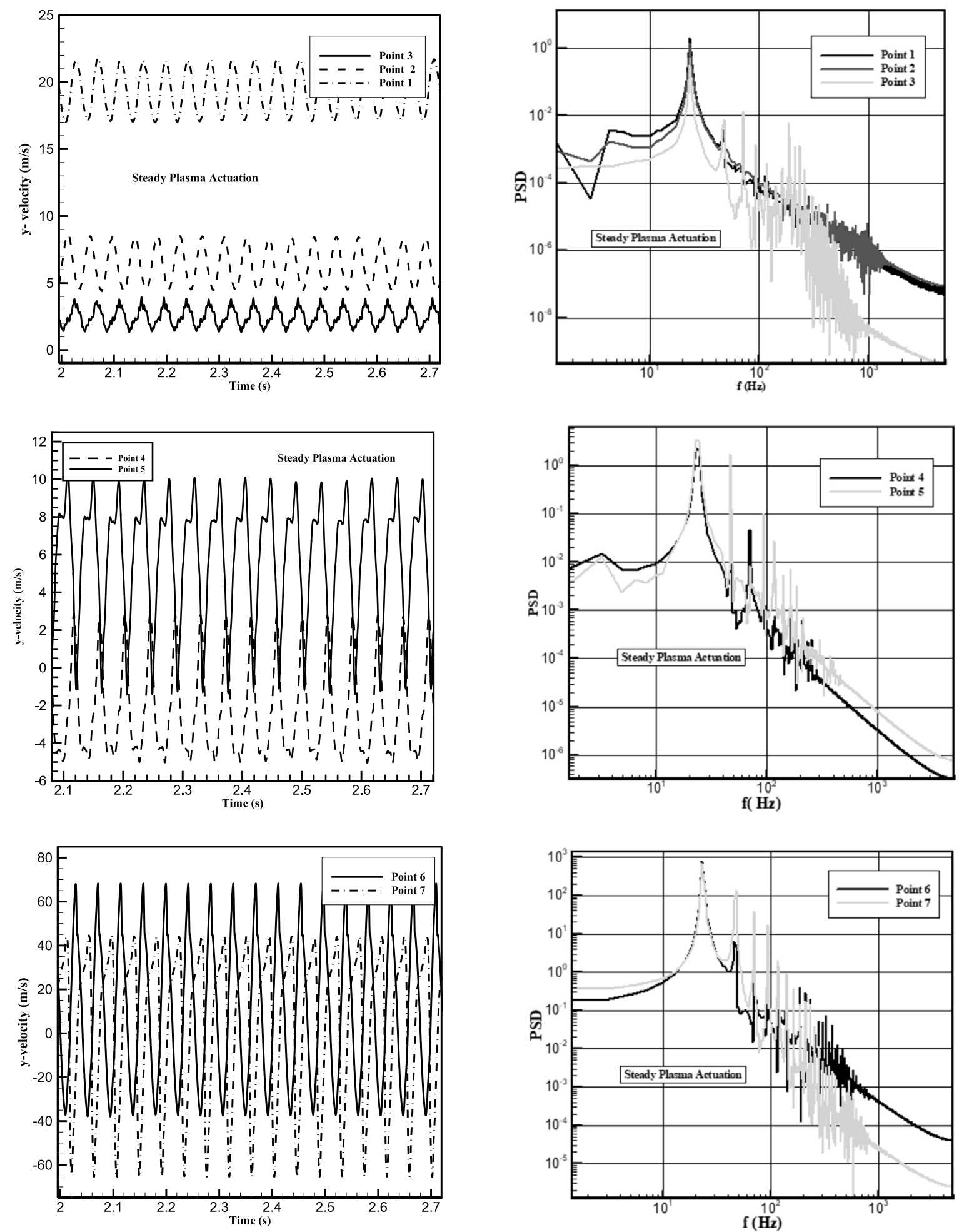

(a)

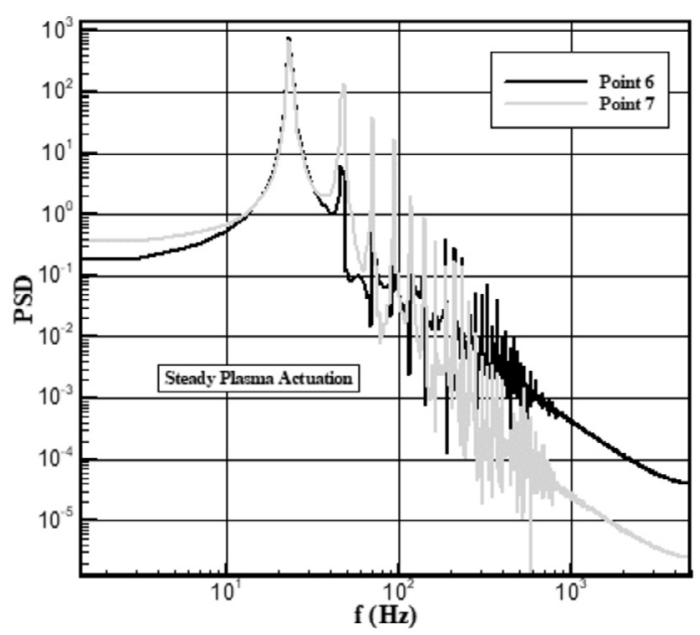

(b)

Fig. 8. Cross-stream velocity component signals at different probe locations with steady plasma actuation: a) time variation; b) power spectra.

frequencies, $f=25 \mathrm{~Hz}$ and $50 \mathrm{~Hz}$. However, the size of secondary vortex near the trailing edge is reduced in these cases in comparison to the base case (Fig. $4 \mathrm{a}, \beta=0$ ). When the burst frequency is increased to $250 \mathrm{~Hz}$, the unsteady actuation was successful in controlling the separation bubble.

Both the steady and the unsteady actuation processes have improved the pressure recovery in the separation region. In this aspect, the steady actuation was slightly better than the unsteady one. This is quite different from the results for laminar separation control where unsteady actuator operation (mostly at $F^{+}=1$ ) has always been found to be better than steady actuator operation. Similar results for controlling turbulent separation bubble were reported by $\mathrm{He}$ et al. [44]. However, this conclusion from the numerical results could be related to the assumption of the two-dimensional flow followed in the present study. Asada and Fuji [38], suggested that important phenomena related to the ef- 

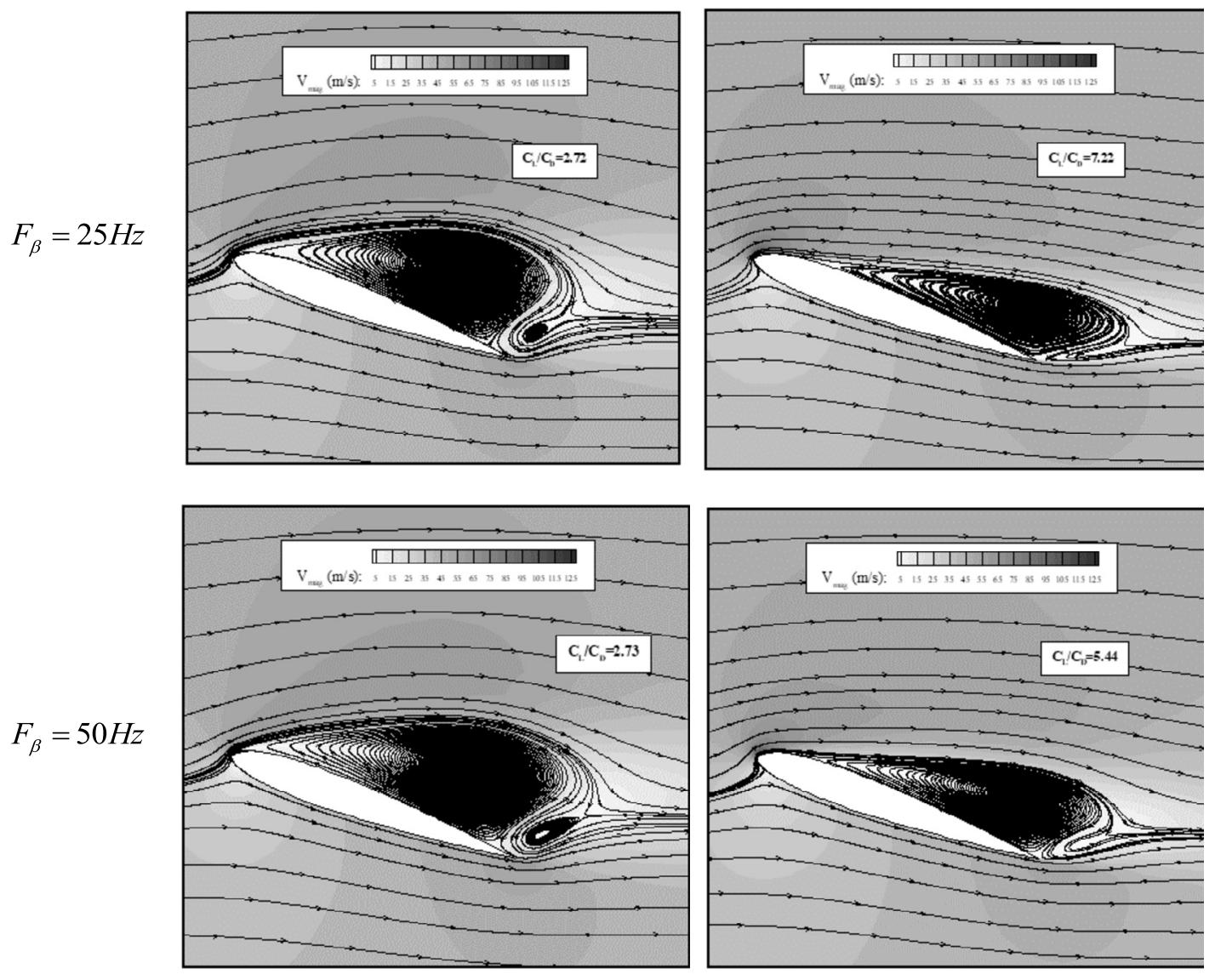

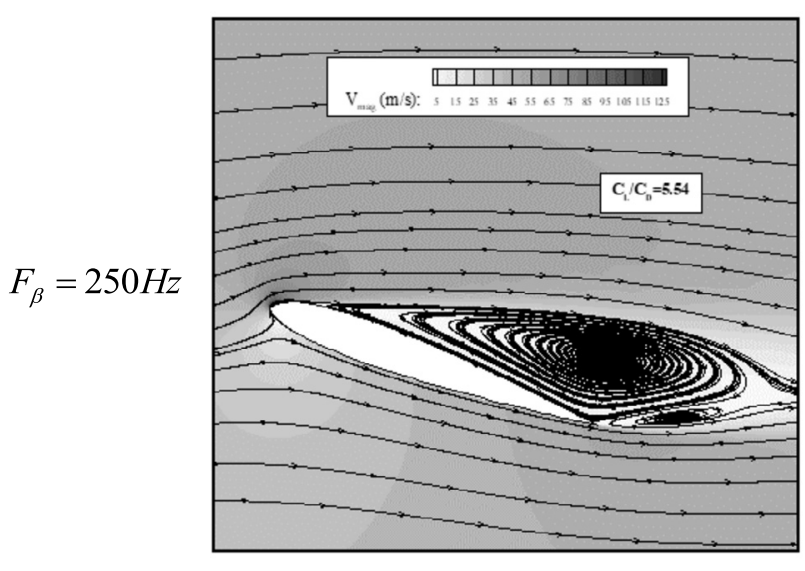

(a)

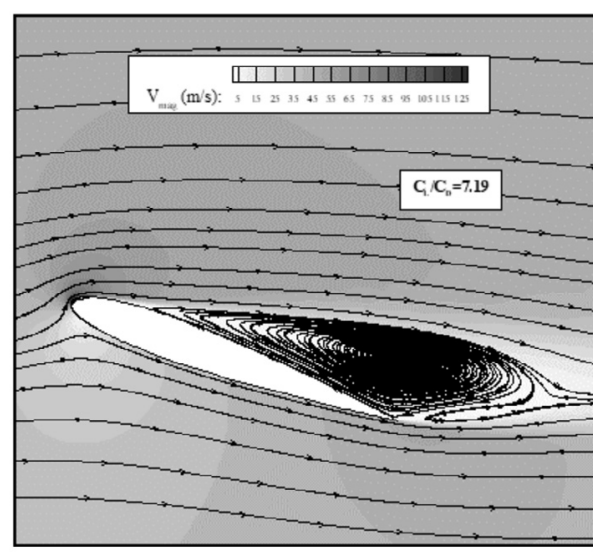

(b)

Fig. 9. Time-averaged velocity contours and streamlines at an angle of attack of $20^{\circ}$ and three burst frequencies for: a) $\beta=10 \%$; b) $\beta=50 \%$.

fect of the burst mode on separation control using plasma actuators may be three-dimensional in nature which will then tend to induce strong longitudinal vortices sometimes observed in the experiments. Obviously, the present two dimensional approach is unable to predict such phenomena, in particular the interaction between transversal vortices and the main longitudinal flow, but the comparison given in Fig. 4b provides some support to expect that three-dimensional effects are small in the present flow geometry.

\subsubsection{Comparison of the steady and unsteady modes: local pressure coefficients}

Mean surface pressure coefficients for the base flow and the steady and unsteady actuator cases are compared in Fig. 10. When the plasma actuator is off, the flow-field around the airfoil separates from the leading edge and the pressure coefficient has a flat distribution over the whole suction side of the airfoil. However, when the steady or unsteady actuation is on, the body force produced by the plasma actuator locally accelerates the ambient air, thus producing a strong low pressure region and thus a sharp decline in the $C p$ level at the actuator position at the leading edge, which is then followed by a rapid pressure recovery towards the trailing edge. The peak value of the pressure coefficient in the presence of the plasma actuator is reduced. For the unsteady cases the largest value of the peak of $C p$ occurred at $F_{\beta}=250 \mathrm{~Hz}$ and $25 \mathrm{~Hz}$, for $\beta=10 \%$ and $50 \%$, respectively. Moreover for $\beta=10 \%$ and $F_{\beta}=250 \mathrm{~Hz}$, the size of the large plateau of $C p$ on the suction surface (a characteristic of the separation side) is significantly 


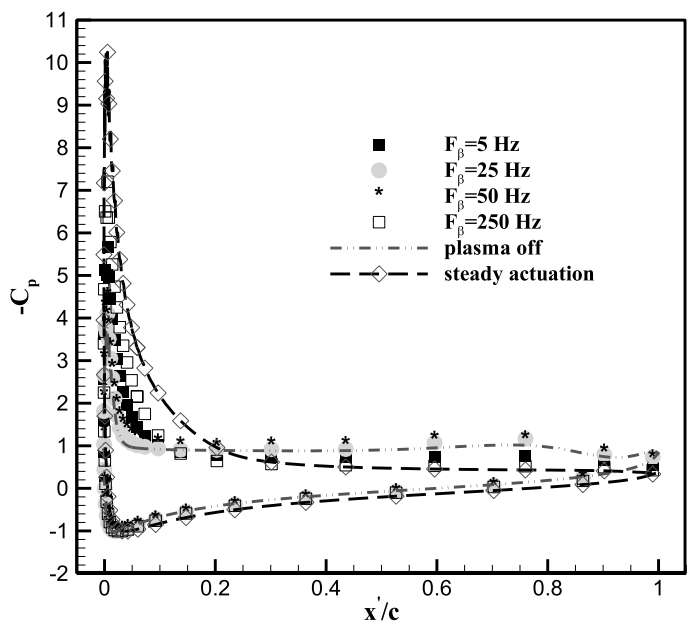

(a)

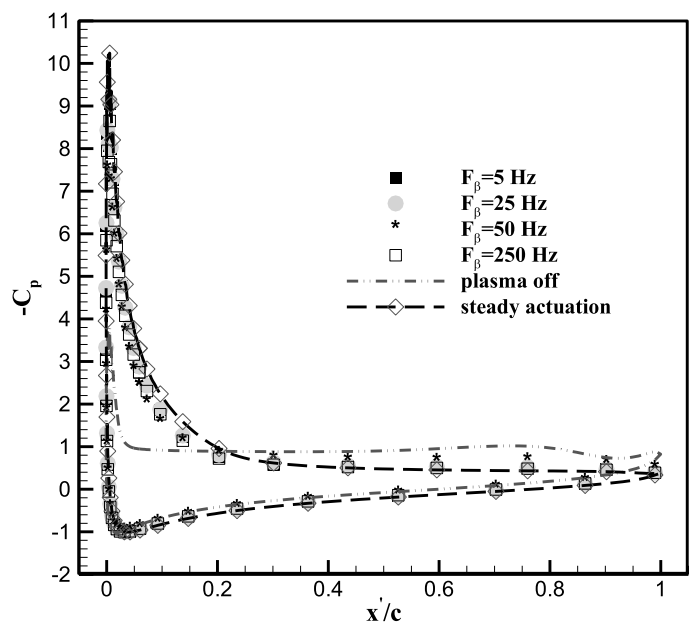

(b)

Fig. 10. Pressure coefficient distribution around the NACA0012 airfoil at an angle of attack of $20^{\circ}$ for steady and unsteady plasma actuation with: a) $\beta=10 \%$; $) \beta=50 \%$.

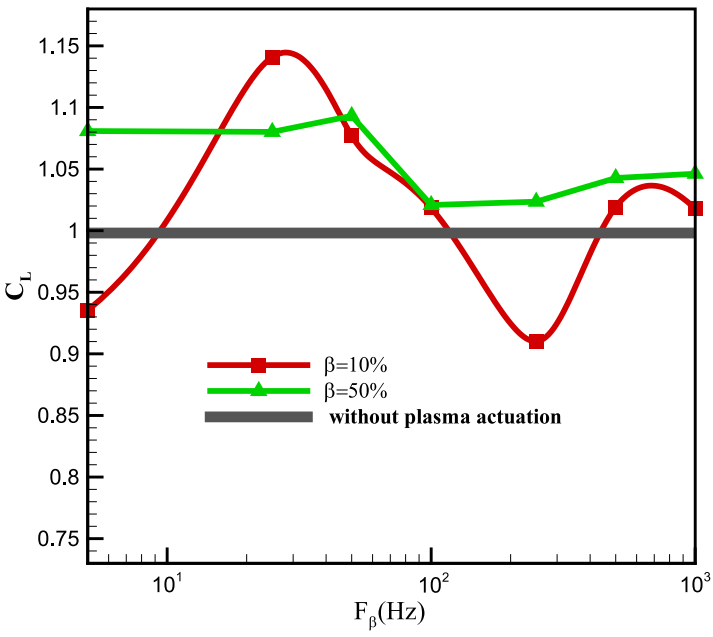

(a)

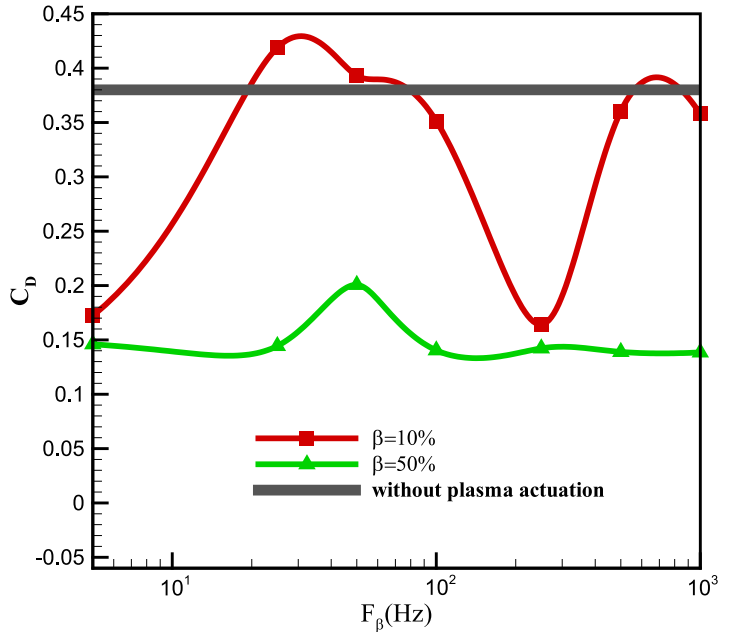

(b)

Fig. 11. Effect of unsteady actuation frequency and burst percentage on: a) lift coefficient; b) drag coefficient.

reduced. Therefore, the lift of the airfoil can be improved. However, this figure shows that the steady actuation (normal mode) is more effective than unsteady actuation (burst mode) on the control of separation in this flow condition.

\subsubsection{Influence of burst conditions on lift and drag coefficients}

In Fig. 11, the effect of two burst ratios, 10\% and 50\%, and different burst frequencies on the lift and drag coefficients is demonstrated. At a burst ratio of $10 \%$, the lift and drag coefficients have experienced a maximum increment of respectively, $12.5 \%$ and $10.2 \%$ at $F_{\beta}=25 \mathrm{~Hz}$. Hence, even duty cycles as low as $10 \%$ are able to increase the lift coefficient. However, at the same burst ratio, when the burst frequency is increased to $250 \mathrm{~Hz}$, both lift and drag are reduced to a minimum by $9.7 \%$ and $21.5 \%$, respectively, compared with the plasma-off simulation. Moreover, it was observed that almost for all the cases the increase in lift was accompanied by a decrease in drag and vice versa. When $\beta=50 \%$, the lift and the drag curves just have an extremum at $F_{\beta}=50 \mathrm{~Hz}$. The results also confirmed that it was possible to obtain the same lift coefficient by consuming less amount of energy (e.g. $F_{\beta}=100 \mathrm{~Hz}$ ).

The predicted lift and drag coefficients for different burst frequencies and two different burst percentages, together with their variance are also summarized in Table 4 . These data show that, for all the cases, the separation point moves downstream when unsteady actuation is on, in comparison to the case without plasma actuation. When the burst frequency is equal to $50 \mathrm{~Hz}$, the oscillation amplitude of the lift and drag (excitation-induced oscillations) is maximum for the two burst ratios, $\beta=10 \%$ and $\beta=50 \%$. This shows that instabilities in the flow were excited, and not damped, by the plasma actuation. When $\beta=50 \%$, the variance of the lift and drag coefficients is seen to be reduced continuously by an increase in the burst frequency, and are much smaller in comparison with the case of $\beta=10 \%$. It should be clear that such an increase in burst ratio causes the plasma actuator to act closer to the steady operation mode, which is more effective in stabilization of the broad band frequencies of the flow (see Fig. 7).

In Table 4, the rows belonging to the frequencies for which optimum or resonance behavior is observed are highlighted for emphasis. Considering the results obtained, the optimum or resonance frequency in unsteady mode of actuation can be interpreted in two ways. As shown by Patel et al. [29] and Asada et al. [27], there exists a frequency for which the actuation voltage controlling the flow in unsteady manner has the smallest (minimum) amplitude (optimum mode). The second viewpoint is that, disregard of the voltage there exists a frequency for which the controlling/disturbing effect of the actuator is more pronounced (resonance mode). 
Table 4

Summary of the influence of the unsteady actuation. $\beta=0 \%$

\begin{tabular}{lllllll}
$\beta=0 \%$ & \multicolumn{1}{l}{} & & & \\
\hline- & $C_{L}$ & $C_{D}$ & $C_{L} / C_{D}$ & $x_{\text {sep }}$ & $\operatorname{Var}_{c l}$ & $\operatorname{Vr}_{c d}$ \\
\hline & 0.9981 & 0.38 & 2.626579 & 0.020022 & 0.005199 & 0.000524 \\
\hline
\end{tabular}

\begin{tabular}{lllllll}
$\beta=10 \%$ & \multicolumn{5}{l}{} \\
\hline$F_{\beta}$ & $C_{L}$ & $C_{D}$ & $C_{L} / C_{D}$ & $x_{s e p}$ & $\operatorname{Var}_{c l}$ & $\operatorname{Var}_{c d}$ \\
\hline 5 & 0.935366 & 0.17213 & 5.434061 & 0.082837 & 0.0008141 & $6.33 \mathrm{E}-05$ \\
25 & 1.140893 & 0.41886 & 2.723805 & 0.03826 & 0.005806389 & 0.0006336 \\
50 & 1.076958 & 0.393098 & 2.739668 & 0.023833 & 0.015185 & 0.001784 \\
100 & 1.018479 & 0.351313 & 2.899062 & 0.026955 & 0.004855 & 0.000209121 \\
250 & 0.91012 & 0.164078 & 5.546874 & 0.1158 & 0.0001029 & $1.37 \mathrm{E}-06$ \\
500 & 1.019219 & 0.36028 & 2.828964 & 0.081117 & $6.15 \mathrm{E}-03$ & 0.0002623 \\
1000 & 1.017927 & 0.358106 & 2.842528 & 0.0329 & 0.006054 & 0.00026 \\
\hline
\end{tabular}

\begin{tabular}{lllllll}
$\beta=50 \%$ & \multicolumn{5}{l}{} \\
\hline$F_{\beta}$ & $C_{L}$ & $C_{D}$ & $C_{L} / C_{D}$ & $x_{\text {sep }}$ & $V a r_{C l}$ & $\operatorname{Var}_{C d}$ \\
\hline 5 & 1.08089 & 0.14598 & 7.40437 & 0.171168 & 0.00103016 & 0.00012035 \\
25 & 1.080187 & 0.1444 & 7.221219 & 0.178534 & 0.00188 & $6.58 \mathrm{E}-05$ \\
50 & 1.09316 & 0.20061 & 5.44918 & 0.14785 & 0.0073944 & 0.004399 \\
100 & 1.02082 & 0.1404 & 7.270798 & 0.168786 & $1.60 \mathrm{E}-06$ & $1.60 \mathrm{E}-06$ \\
250 & 1.023525 & 0.142204 & 7.197572 & 0.164954 & $5.84 \mathrm{E}-05$ & $1.74 \mathrm{E}-06$ \\
500 & 1.042744 & 0.138942 & 7.504887 & 0.172284 & $7.05 \mathrm{E}-07$ & $4.18 \mathrm{E}-08$ \\
1000 & 1.04623 & 0.138444 & 7.557063 & 0.173402 & $8.00 \mathrm{E}-07$ & $2.40 \mathrm{E}-08$ \\
\hline
\end{tabular}

\begin{tabular}{|c|c|c|c|c|c|c|}
\hline- & $C_{L}$ & $C_{D}$ & $C_{L} / C_{D}$ & $x_{\text {sep }}$ & $\mathrm{Var}_{c l}$ & $V a r_{c d}$ \\
\hline & 1.16795 & 0.118068 & 9.892181 & 0.23079 & - & - \\
\hline
\end{tabular}

We should note that, although the unsteady actuation employed in the current study was not found to be more efficient that the steady actuation, a careful analysis of our results confirms the recently reported data of [30] in the sense that at a burst frequency of $F^{+}=1$, the major flow structures are excited in a resonant manner (the variance of the oscillations were maximum as well as the lift and drag increments) and for $F^{+} \geq 5.75$, best aerodynamic efficiencies were obtained (similar to $[27,30]$ ). In addition, our results show that at $F_{\beta}=5 \mathrm{~Hz}$ the aerodynamic efficiency was already enhanced. This observation might be related to the fact that this low frequency is of the order of the frequency of the shear layer here predicted, and so it is sufficient for exciting the roll up vorticities at the leading edge and thus promoting separation control.

\subsubsection{Influence of bust conditions on dominant frequencies}

To further clarify the effect of the burst frequency and burst ratio for the case of unsteady actuation, Fig. 12 shows the power spectra for the temporal variation of the cross-stream velocity ( $y$-component) at different probe locations (indicated in Fig. 5) for an angle of attack of $20^{\circ}$. These cases correspond to the frequencies $F_{\beta}=50 \mathrm{~Hz}$ and $250 \mathrm{~Hz}$. PSD of the $y$-component of the velocity at points $1-3$ correspond to unsteady flow in the shear layer. When the burst ratio is 50\% the peaks of the flow instabilities are shifted to lower frequencies, however the PSD shows several weak oscillations in a frequency range larger than $52 \mathrm{~Hz}$. In the separation zone and the shedding region, the frequency bump is slightly shifted in a way that, in the separation region, the major frequency becomes locked at a value around $52 \mathrm{~Hz}$ (resonance situation). When the frequency of the actuation is increased to $250 \mathrm{~Hz}$, the magnitude of the power spectra density at those locations is reduced and the peak of the frequency map for the vortex shedding frequency is locked at the imposed excitation frequency, moreover all fluctuations with a frequency range below $250 \mathrm{~Hz}$ are filtered (optimum case). In this case, the interaction and resonance between shear layer and wake vortex shedding results in optimum control authority.

In contrast, when the burst ratio is $10 \%$ and burst frequency is equal to $F_{\beta}=50 \mathrm{~Hz}$, the power spectra exhibit strong peaks at the burst frequency and its harmonics in the range $24.66 \mathrm{~Hz}$ to $100.76 \mathrm{~Hz}$, an indication that the plasma actuator is clearly contributing to the production of turbulence. This may explain the drag increase in unsteady actuation at this frequency, in comparison to the steady actuation. Essentially, the coexistence of fluid motions with different frequencies of movement in the wake, and thus the momentum and energy exchange among these structures, could result in disorganization of the flow motions and extra losses. However, the flow in the shedding region and separation bubble is more organized and regulated, since the low amplitude and high frequencies in the flow disappear in this case. When the burst frequency is increased to $250 \mathrm{~Hz}$, the FFT analysis shows that the motions at lower frequency in the flow field are successfully suppressed (similar behavior to the case of $\beta=50 \%$ ).

We might hypothesize that the efficiency of the unsteady plasma actuation could be dependent on different parameters, including the electrical and geometrical characteristics of the DBD and also the characteristic of the regime of the flow to be controlled [45]. For example, if the frequency of actuation does not match the natural frequencies of the flow, either the actuation is not in the optimum mode or the perturbation injected by the plasma actuator is destabilizing the flow in a negative way. On the other hand, when the voltage amplitude is considerable and the steady actuation already controls the flow, the unsteady actuation with large forcing amplitude may induce unwanted oscillations in the flow which will reduce the efficiency of flow control. Also, efficiency of momentum injection and mixing will be reduced when the free stream velocity is large or the boundary layer is too energetic (turbulent boundary layer). Thus when the flow velocity is higher, the flow structure is less receptive to the unsteady disturbances produced by the plasma actuator and the effect of unsteady actuation would tend to decrease [46]. For example, Asghar et al. [47] and Sulaiman et al. [48] have shown that the Reynolds number could be used as a scaling parameter for considering the effectiveness of the DBD plasma actuator. Moreover, the configuration of the DBD may influence the potential of the actuator for flow control. Since steady and unsteady actuations imply different approaches for controlling the flow, the optimum configuration 
a)

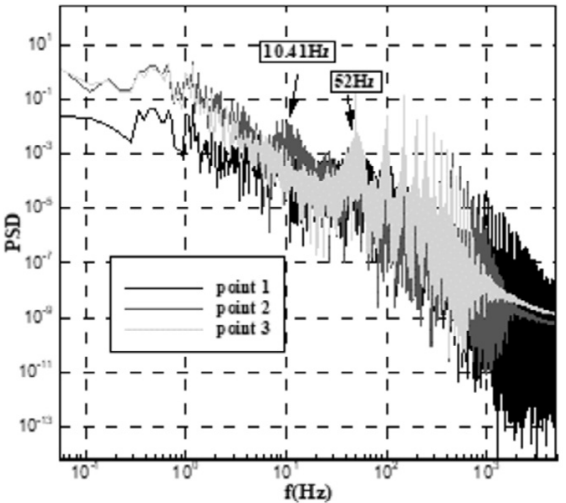

b)

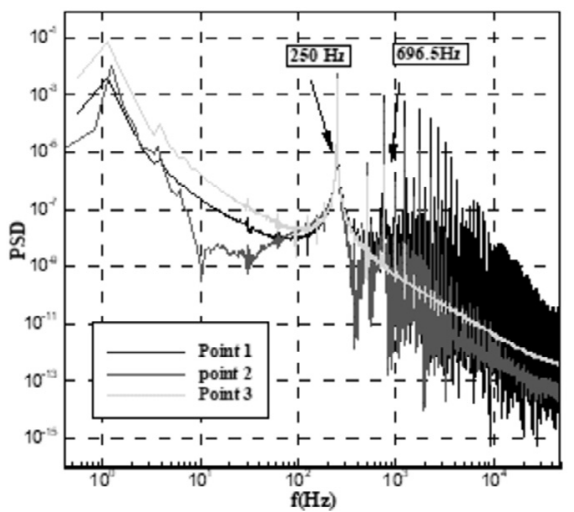

c)

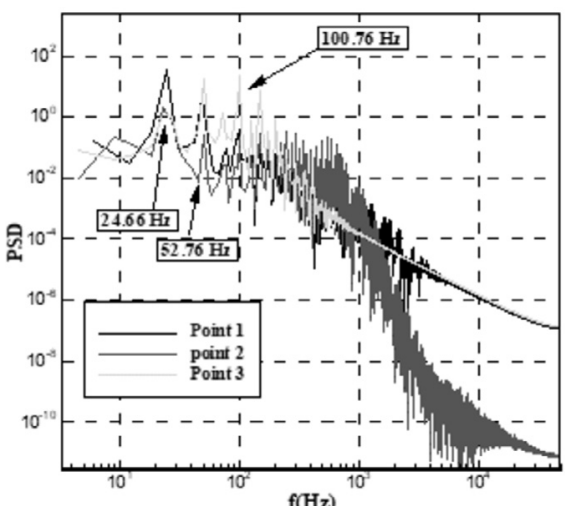

d)

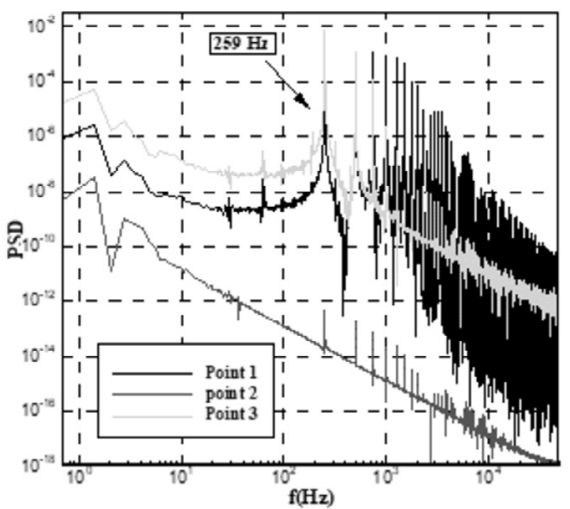

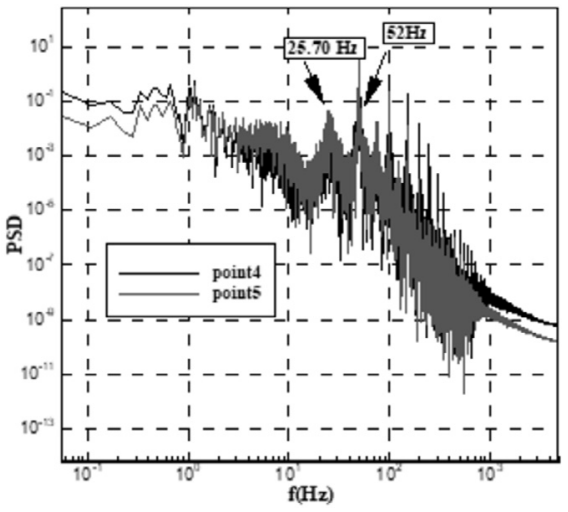
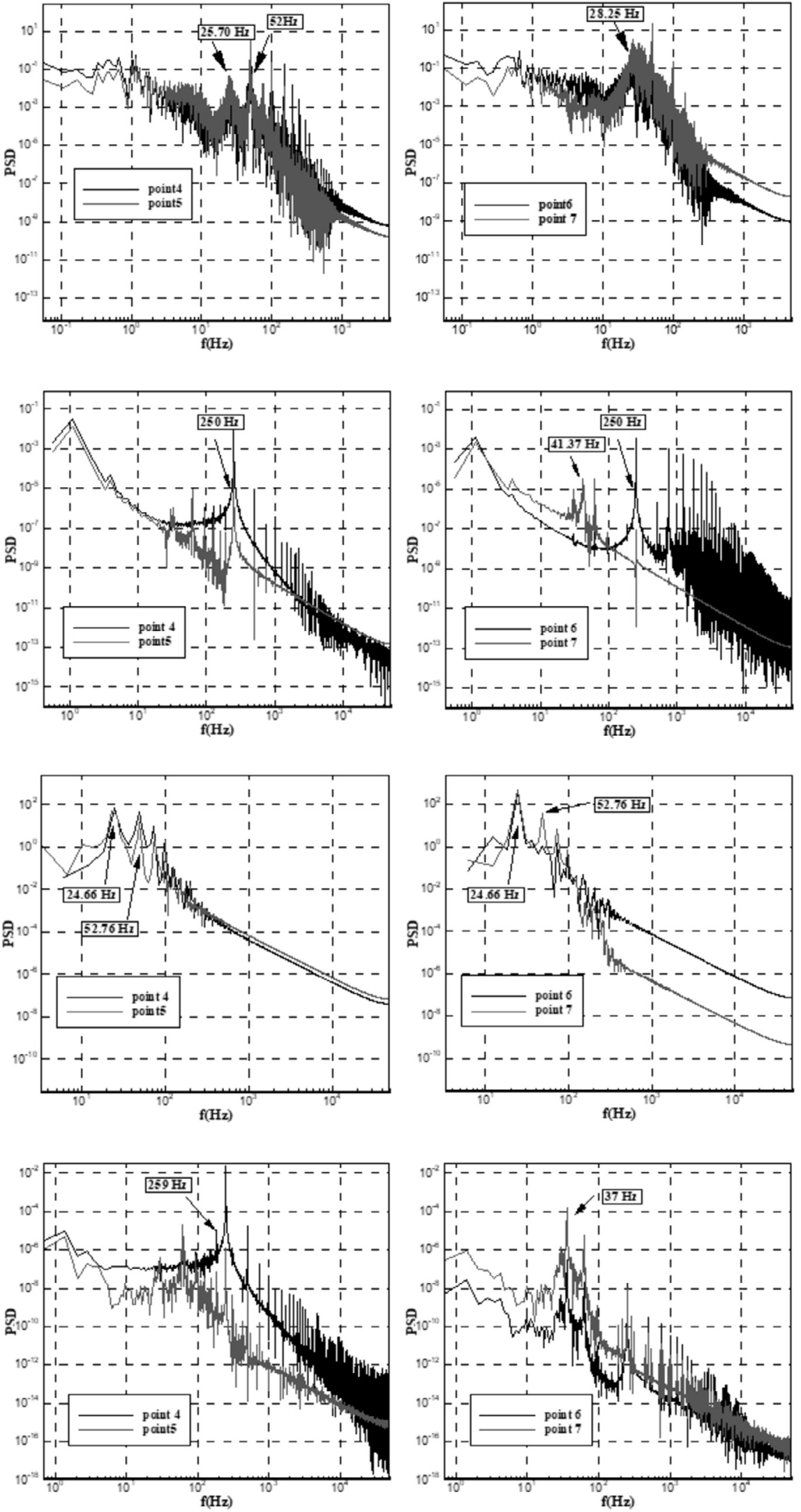

Fig. 12. Power spectra of $y$-velocity component for a) $F_{\beta}=50 \mathrm{~Hz}, \beta=50 \%$; b) $F_{\beta}=250 \mathrm{~Hz}, \beta=10 \%$; $) F_{\beta}=50 \mathrm{~Hz}, \beta=10 \%$; d) $F_{\beta}=250 \mathrm{~Hz}, \beta=10 \%$. 
(e.g. DBD position) might work in one case and might fail to control the flow in other cases.

\section{Conclusions}

A numerical study was conducted to investigate and compare the effect of steady and unsteady actuation modes for controlling flow at relatively high Reynolds number $\left(\operatorname{Re}=10^{6}\right)$. A new phenomenological model was used to simulate the effect of the plasma actuator. It can be concluded that the DBD plasma actuator offers a relatively effective means for controlling the stalled airfoil flow. The large separation region on stalled airfoil was not completely removed by the plasma actuator in the present configuration of the DBD, however the size of the time-averaged separation bubble was greatly reduced. The flow structure was found to be sensitive to the frequency of burst modulation.

The results showed that in the case of unsteady actuation, the burst frequency and burst ratio are crucial parameters influencing the flow capability of the actuators. This actuation mode consumes less power and achieves the desirable control effect. Burst frequencies near the natural frequencies of the system were able to excite the flow structure in a resonance mode. In this case, the instabilities and internal losses in the flow structure tend to increase (resonance mode), which limits the efficiency of the actuators. However, higher frequencies of around of $250 \mathrm{~Hz}$ show better flow control. In this case the flow frequencies become less energetic, implying that the internal process of exchanging momentum and energy among the flow structures becomes more constrained and thus flow losses are reduced. This observation also confirms that with proper frequencies of excitation, the flow structure can be well rearranged and flow losses can be reduced.

The computational study presented here clearly confirmed the effect of the burst frequency and duty cycle percentage on controlling and regulating the flow structure. However, the unsteady actuation employed here showed lower efficiency in comparison to the steady actuation mode. The reason could be ascribed to several factors, considering the mechanism of flow control by unsteady plasma actuators. To further investigate the flow control mechanism, 3D simulations need to be done using more sophisticated turbulence models (e.g. LES models) to eliminate the averaging procedure of the RANS models and also to better simulate the dynamics of the three-dimensional vortex structures.

\section{Conflict of interest statement}

There is no conflict of interest to be stated.

\section{Acknowledgements}

The research work was supported by FCT Project PTDC/EMSENE/5742/2014 UNDULANT-Next (Unsteady boundary Layer flow control using plasma actuators of Next generation), part of the work was also supported by C-MAST (Center for Mechanical and Aerospace Science and Technology) funded by Compete 2020 and FCT project UID/EMS/00151/2013.

\section{References}

[1] R. Duvigneau, M. Visonneau, Simulation and optimization of stall control for an airfoil with a synthetic jet, Aerosp. Sci. Technol. 10 (2006).

[2] B. Yagiz, O. Kandil, Y.V. Pehlivanoglu, Drag minimization using active and passive flow control techniques, Aerosp. Sci. Technol. 17 (2012) 21-31.

[3] A. Rezaei, A. Ramiar, A.A. Ranjbar, M. Abdollahzadeh, A. Kianifar, Influence of repetitive laser pulse energy depositions on supersonic flow over a sphere, cone and oblate spheroid, Aerosp. Sci. Technol. 76 (2018) 72-81, https:/ doi.org/10.1016/j.ast.2018.02.005.
[4] S. Das, M. Abdollahzadeh, J. Pascoa, A. Dumas, M. Trancossi, Numerical modeling of Coanda effect in a novel propulsive system, Int. J. Multiphys. 8 (2014) $181-201$.

[5] A. Ghaedamini Harouni, Flow control of a boundary layer ingesting serpentine diffuser via blowing and suction, Aerosp. Sci. Technol. 39 (2014) 472-480.

[6] M. Dghim, M. Ferchichi, R.E. Perez, M. BenChiekh, Near wake development of a wing tip vortex under the effect of synthetic jet actuation, Aerosp. Sci. Technol. 54 (2016) 88-107.

7] G. Ilieva, J. Páscoa, A. Dumas, M. Trancossi, MAAT - promising innovative design and green propulsive concept for future airship's transport, Aerosp. Sci. Technol. 35 (2014) 1-14, https://doi.org/10.1016/j.ast.2014.01.014.

[8] M. Abdollahzadeh, J.C. Páscoa, P.J. Oliveira, Two-dimensional numerical modeling of interaction of micro-shock wave generated by nanosecond plasma actuators and transonic flow, J. Comput. Appl. Math. 270 (2013) 401-416, https:// doi.org/10.1016/j.cam.2013.12.030.

[9] Y.-C. Cho, W. Shyy, Adaptive flow control of low-Reynolds number aerodynamics using dielectric barrier discharge actuator, Prog. Aerosp. Sci. 47 (2011) 495-521, https://doi.org/10.1016/j.paerosci.2011.06.005.

[10] J. Morgado, M. Abdollahzadeh, M.A.R. Silvestre, J.C. Páscoa, High altitude propeller design and analysis, Aerosp. Sci. Technol. 45 (2015) 398-407.

[11] M. Abdollahzadeh, F. Rodrigues, J.C. Pascoa, P.J. Oliveira, Numerical design and analysis of a multi-DBD actuator configuration for the experimental testing of ACHEON nozzle model, Aerosp. Sci. Technol. 41 (2015) 259-273.

[12] M. Abdollahzadeh, J.C. Pascoa, P.J. Oliveira, Implementation of the classical plasma-fluid model for simulation of dielectric barrier discharge (DBD) actuators in OpenFOAM, Comput. Fluids 128 (2016) 77-90.

[13] M. Kotsonis, S. Ghaemi, Experimental and numerical characterization of a plasma actuator in continuous and pulsed actuation, Sens. Actuators A, Phys. 187 (2012) 84-94, https://doi.org/10.1016/j.sna.2012.08.002.

[14] J.A. Ekaterinaris, Prediction of active flow control performance on airfoils and wings, Aerosp. Sci. Technol. 8 (2004) 401-410.

[15] A. Seifert, D. Greenblatt, I.J. Wygnanski, Active separation control: an overview of Reynolds and Mach numbers effects, Aerosp. Sci. Technol. 8 (2004) 569-582.

[16] M.L. Post, T.C. Corke, Separation control on high angle of attack airfoil using plasma actuators, AIAA J. 42 (2004) 2177-2184, https://doi.org/10.2514/1.2929.

[17] A. Abdoli, I. Mirzaee, A. Anvari, N. Purmahmod, Simulation of body force field effects on airfoil separation control and optimization of plasma actuator, J. Phys. D, Appl. Phys. 41 (2008) 175204, https://doi.org/10.1088/0022-3727/41/ $17 / 175204$.

[18] Duchmann, Boundary-Layer Stabilization with Dielectric Barrier Discharge Plasmas for Free-Flight Application, PhD thesis, Technischen Universität Darmstadt, 2013.

[19] M.D. Chernyshev, Sergey L. Gamirullin, V.Y. Khomich, A.P. Kuryachii, V.M. Litvinov, S.V. Manuilovich, S.I. Moshkunov, et al., Electrogasdynamic laminar flow control on a swept wing, Aerosp. Sci. Technol. 59 (2016) 155-161.

[20] R. Erfani, T. Erfani, S.V. Utyuzhnikov, K. Kontis, Optimisation of multiple encapsulated electrode plasma actuator, Aerosp. Sci. Technol. 26 (2013) 120-127.

[21] D. Tsubakino, Y. Tanaka, Effective Layout of Plasma Actuators for a Flow Separation Control on a Wing, AIAA, 2007, p. 474.

[22] M. Abdollahzadeh, J.C. Páscoa, P.J. Oliveira, Numerical modeling of boundary layer control using dielectric barrier discharge, in: Conferência Nac. Em Mecânica Dos Fluidos, Termodinâmica E Energ. MEFTE, vol. 61, 2012, pp. 1-10.

[23] N. Balcon, N. Benard, Y. Lagmich, J.-P. Boeuf, G. Touchard, E. Moreau, Positive and negative sawtooth signals applied to a DBD plasma actuator - influence on the electric wind, J. Electrost. 67 (2009) 140-145, https://doi.org/10.1016/j. elstat.2009.01.019.

[24] M. Kotsonis, S. Ghaemi, Performance improvement of plasma actuators using asymmetric high voltage waveforms, J. Phys. D, Appl. Phys. 45 (2012) 045204, https://doi.org/10.1088/0022-3727/45/4/045204.

[25] T. Kimura, K. Udagawa Takashima, H. Yamasaki, Experimental study of DBD plasma actuator with combination of $\mathrm{AC}$ and nanosecond pulse voltage, Electr. Eng. Jpn. 185 (2013) 21-29, https://doi.org/10.1002/eej.22292.

[26] B. Goeksel, D. Greenblatt, I. Rechenberg, Y. Kastantin, C.N. Nayeri, C.O Paschereit, Pulsed plasma actuators for active flow control at MAV Reynolds numbers, in: Active Flow Control, in: Notes Numer. Fluid Mech. Multidiscip. Des., vol. 95, Springer, 2007, pp. 42-55.

[27] K. Asada, Y. Ninomiya, A. Oyama, K. Fujii, Airfoil flow experiment on the duty cycle of DBD plasma actuator, in: AIAA 2009-531, 2009, pp. 1-14.

[28] P. Audier, D. Hong, A. Leroy, Unsteady forcing of a post-stall flow over a NACA0012 airfoil by a surface DBD actuator, in: AIAA 2012-3052, 2012, pp. 1-9.

[29] M.P. Patel, T.T. Ng, S. Vasudevan, T.C. Corke, M. Post, T.E. McLaughlin, et al., Scaling effects of an aerodynamic plasma actuator, J. Aircr. 45 (2008) 223-236, https://doi.org/10.2514/1.31830.

[30] A.A. Sidorenko, B.V. Postnikov, A.D. Budovsky, D.V. Roupassov, I.N. Zavialov, N.D. Malmuth, et al., Pulsed discharge actuators for rectangular wing separation control, in: 45th AIAA Aerosp. Sci. Meet. Exhib., 2007, p. 941.

[31] K. Mitsuo, S. Watanabe, T. Atobe, H. Kato, U. Tatsuro, M. Tanaka, et al., Lift enhancement of a pitching airfoil in dynamic stall by DBD plasma actuators, in: 51st AIAA Aerosp. Sci. Meet. Incl. New Horizons Forum Aerosp. Expo, 2013, p. 1119. 
[32] N. Benard, J. Jolibois, E. Moreau, Lift and drag performances of an axisymmetric airfoil controlled by plasma actuator, J. Electrost. 67 (2009) 133-139, https:// doi.org/10.1016/j.elstat.2009.01.008.

[33] N. Benard, E. Moreau, Capabilities of the dielectric barrier discharge plasma actuator for multi-frequency excitations, J. Phys. D, Appl. Phys. 43 (2010) 145201, https://doi.org/10.1088/0022-3727/43/14/145201.

[34] Y.E. Akansu, F. Karakaya, a. Sanlısoy, Active control of flow around NACA 0015 airfoil by using DBD plasma actuator, EPJ Web Conf. 45 (2013) 01008, https:// doi.org/10.1051/epjconf/20134501008.

[35] A.J. Lombardi, P.O. Bowles, T.C. Corke, Closed-loop dynamic stall control using a plasma actuator, AIAA J. 51 (2013) 1130-1141, https://doi.org/10.2514/1. J051988.

[36] M. Abdollahzadeh, J.C. Pascoa, P.J. Oliveira, Modified split-potential model for modeling the effect of DBD plasma actuators in high altitude flow control, Curr. Appl. Phys. 14 (2014) 1160-1170, https://doi.org/10.1016/j.cap.2014.05.016.

[37] H.G. Weller, G. Tabor, H. Jasak, C. Fureby, A tensorial approach to computational continuum mechanics using object-oriented techniques, Comput. Phys. 12 (1998) 620, https://doi.org/10.1063/1.168744.

[38] K. Asada, K. Fujii, Computational analysis of unsteady flow-field induced by plasma actuator in burst mode, in: AIAA 2010-5090, 2010, pp. 1-12.

[39] E. Garnier, P.Y. Pamart, J. Dandois, P. Sagaut, Evaluation of the unsteady RANS capabilities for separated flows control, Comput. Fluids 61 (2012) 39-45, https://doi.org/10.1016/j.compfluid.2011.08.016.

[40] F. Menter, T. Esch, Elements of industrial heat transfer prediction, in: Proc. 16th Brazilian Congr. Mech. Eng., 2001, pp. 117-127.
[41] W. Haase, M. Braza, A. Revell, DESider - A European Effort on Hybrid RANS-LES Modeling, Notes Numer. Fluid Mech. Multidisciplinary Des., vol. 103, Springer 2009.

[42] C.L. Ladson, Effects of Independent Variation of Mach and Reynolds Numbers on the Low-Speed Aerodynamic Characteristics of the NACA 0012 Airfoil Section, Hampton, VA, United States, 1988.

[43] D. Greenblatt, T. Schneider, C.Y. Schüle, Mechanism of flow separation contro using plasma actuation, Phys. Fluids 24 (2012) 077102, https://doi.org/10.1063/ 1.4733399.

[44] C. He, T.C. Corke, N. Dame, Numerical and experimental analysis of plasma flow control over a hump model electrode AC voltage source, in: AIAA, 2007, pp. 1-16.

[45] Y.-C. Cho, W. Shyy, Adaptive control of low-Reynolds number aerodynamics in uncertain environments: Part 1. Disturbance regimes and flow characteristics, Comput. Fluids 86 (2013) 582-596, https://doi.org/10.1016/j.compfluid. 2013.04.013.

[46] B. Göksel, D. Greenblatt, I. Rechenberg, Y. Singh, C.N. Nayeri, C.O. Paschereit, Pulsed plasma actuators for separation flow control, in: Conf. Turbul. Interact. TI2006, Porquerolles, France, 2006, pp. 1-4.

[47] A. Asghar, Eric J. Jumper, Thomas C. Corke, On the use of Reynolds number as the scaling parameter for the performance of a plasma actuator in a weakly compressible flow, in: AIAA, 2006, pp. 2006-2021.

[48] T. Sulaiman, H. Aono, E. Agency, S. Sekimoto, M. Anyoji, T. Nonomura, et al., Effect of burst frequency and Reynolds number on flow control authority of DBD plasma actuator on NACA0012 airfoil, in: AIAA 52nd Aerosp. Sci. Meet. 2014, pp. 1-21. 